Article

\title{
An FDTD Study of Errors in Magnetic Direction Finding of Lightning Due to the Presence of Conducting Structure Near the Field Measuring Station
}

\author{
Yosuke Suzuki ${ }^{1}$, Shohei Araki ${ }^{1}$, Yoshihiro Baba ${ }^{1, *}$, Toshihiro Tsuboi ${ }^{2}$, Shigemitsu Okabe ${ }^{2}$ \\ and Vladimir A. Rakov ${ }^{3,4}$ \\ 1 Department of Electrical Engineering, Doshisha University, Kyoto 610-0321, Japan; \\ lens_chloe@yahoo.co.jp (Y.S.); duq0304@mail4.doshisha.ac.jp (S.A.) \\ 2 TEPCO Research Institute, Tokyo Electric Power Company, Yokohama 230-8510, Japan; \\ tsuboi.toshihiro@tepco.co.jp (T.T.); okabe.s@tepco.co.jp (S.O.) \\ 3 Department of Electrical and Computer Engineering, University of Florida, Gainesville, FL 32611-6130, USA; \\ rakov@ece.ufl.edu \\ 4 Institute of Applied Physics, Russian Academy of Sciences, Nizhny Novgorod 603950, Russia \\ * Correspondence: ybaba@mail.doshisha.ac.jp; Tel.: +81-774-65-6352
}

Academic Editors: Vernon Cooray and Farhad Rachidi

Received: 3 June 2016; Accepted: 12 July 2016; Published: 15 July 2016

\begin{abstract}
Lightning electromagnetic fields in the presence of conducting (grounded) structure having a height of $60 \mathrm{~m}$ and a square cross-section of $40 \mathrm{~m} \times 40 \mathrm{~m}$ within about $100 \mathrm{~m}$ of the observation point are analyzed using the 3D finite-difference time-domain (FDTD) method. Influence of the conducting structure on the two orthogonal components of magnetic field is analyzed, and resultant errors in the estimated lightning azimuth are evaluated. Influences of ground conductivity and lightning current waveshape parameters are also examined. When the azimuth vector passes through the center of conducting structure diagonally (e.g., azimuth angle is $45^{\circ}$ ) or parallel to its walls (e.g., azimuth angle is $0^{\circ}$ ), the presence of conducting structure equally influences $H_{x}$ and $H_{y}$, so that $H_{x} / H_{y}$ is the same as in the absence of structure. Therefore, no azimuth error occurs in those configurations. When the conducting structure is not located on the azimuth vector, the structure influences $H_{x}$ and $H_{y}$ differently, with the resultant direction finding error being greater when the structure is located closer to the observation point.
\end{abstract}

Keywords: lightning locating system; magnetic direction finding; lightning electromagnetic fields; finite-difference time-domain (FDTD) method

\section{Introduction}

Two vertical and orthogonal loops, each measuring the magnetic field from a given vertical radiator, can be used to obtain the direction to the source; that is, as a magnetic field direction finder (DF) [1]. This is the case because the output voltage of a given loop is proportional to the cosine of the angle between the magnetic field vector and the normal vector to the plane of the loop. Therefore, the ratio of the two signals from the loops is proportional to the tangent of the angle between the normal vector to the plane of one of the loops and direction to the source.

Cross-loop magnetic DFs used for lightning detection can be divided into two general types: narrow-band DFs and gated wideband DFs. In both cases, the direction-finding technique involves an implicit assumption that the radiated electric field is oriented vertically and the associated magnetic field is oriented horizontally and perpendicular to the propagation path. 
Narrow-band DFs have been used to detect distant lightning since the 1920s [2,3]. They generally operate in a narrow frequency band with the center frequency in the $5-10 \mathrm{kHz}$ range. A major disadvantage of narrow-band DFs is that for lightning at distances less than about $200 \mathrm{~km}$, those DFs have inherent azimuthal errors, called polarization errors [4,5]. These errors are caused by the detection of magnetic field components from non-vertical channel sections, whose magnetic field lines form circles in a plane perpendicular to the non-vertical channel section. To overcome the problem of large polarization errors at relatively short ranges inherent in the operation of narrow-band DFs, gated wideband DFs were developed in the early 1970s [6]. Direction finding is accomplished by sampling two orthogonal components of the initial peak of the return-stroke magnetic field, that peak being radiated from the bottom hundred meters or so of the channel in the first microseconds of the return stroke. Since the bottom of the channel tends to be straight and vertical, the magnetic field lines form circles in a horizontal plane. The operating bandwidth of the gated wideband DF is typically from a few kilohertz to about $500 \mathrm{kHz}$. Gated wideband DFs, as well as narrow-band DFs, are susceptible to site errors, which are caused by the presence of unwanted magnetic fields due to non-flat terrain and to nearby conducting structures being excited to radiate by the incoming lightning fields.

In multiple-station lightning locating systems (LLSs), such as the U.S. National Lightning Detection Network (NLDN), the direction to lightning is estimated on the basis of the ratio of the peaks of magnetic fields measured by two orthogonal loop antennas, and the lightning location is estimated using the directions reported by multiple pairs of loop antennas.

In countries like Japan, there are often mountains and tall conducting structures near magnetic direction finding stations. As a result, the direction (azimuth) estimated from two orthogonal magnetic fields may be influenced by the presence of mountain and/or conducting structures.

In this paper, lightning electromagnetic fields in the presence of conducting (grounded) structure having a height of $60 \mathrm{~m}$ and a square cross-section of $40 \mathrm{~m} \times 40 \mathrm{~m}$ within about $100 \mathrm{~m}$ of the field measuring point are calculated using the 3D finite-difference time-domain (FDTD) method [7]. Influence of the presence of conducting structure on lightning magnetic fields at the observation point is studied, and the resultant direction (azimuth) errors are discussed. Influences of ground conductivity and lightning current waveshape parameters are also studied. The influence (variation) of structure height is not considered in this paper.

\section{Methodology}

Figure 1a shows the FDTD simulation model of a vertical lightning channel above flat ground. The working volume of $12 \mathrm{~km} \times 12 \mathrm{~km} \times 11 \mathrm{~km}$ is divided uniformly into $20 \mathrm{~m} \times 20 \mathrm{~m} \times 20 \mathrm{~m}$ cubic cells. The time increment is set to $38.1 \mathrm{~ns}$, which fulfills the Courant stability condition. The ground thickness (between the ground surface and the bottom absorbing boundary condition plane) is set to $1 \mathrm{~km}$, and the ground conductivity is set to a value in the range from 0.1 to $1000 \mathrm{mS} / \mathrm{m}$ or to $\infty$ (perfect conductor). The $x z$ plane and the $y z$ plane passing through the source (see Figure 1) are set to be magnetic walls, and other boundaries are each represented by Liao's second-order absorbing boundary condition [8]. Magnetic wall in the FDTD simulation is represented by forcing the tangential magnetic field components on the wall surface to zero. This makes the working volume shown in Figure 1a equivalent to that shown in Figure 1b. The lightning channel is represented by a vertical phased-current-source array [9], which is located at $(x, y)=(0,0)$ of the working volume. The array is activated so as to simulate the current distribution predicted by the transmission-line (TL) model [10]. The return-stroke current waveform is represented using the sum of the Heidler function [11] and a double-exponential function, given as follows:

$$
I(0, t)=\frac{I_{01}}{\eta} \frac{\left(t / \tau_{1}\right)^{2}}{\left(t / \tau_{1}\right)^{2}+1} \exp \left(-\frac{t}{\tau_{2}}\right)+I_{02}\left[\exp \left(-\frac{t}{\tau_{3}}\right)-\exp \left(-\frac{t}{\tau_{4}}\right)\right]
$$


This function reproduces the observed concave rising portion of typical lightning current waveform. In the present simulations, $I_{01}=11 \mathrm{kA}, I_{02}=7.5 \mathrm{kA}, \tau_{2}=5.0 \mu \mathrm{s}, \tau_{3}=100 \mu \mathrm{s}$, and $\tau_{4}=6.0 \mu \mathrm{s}$. For representing $10 \%$-to- $90 \%$ risetime of $0.5,1$, and $3 \mu \mathrm{s}, \tau_{1}=0.305,0.75$, and $4.026 \mu \mathrm{s}$, and $\eta=0.836$, 0.717 , and 0.34585 , respectively. The return-stroke speed is set to $1.5 \times 10^{8} \mathrm{~m} / \mathrm{s}$ (one half of the speed of light).

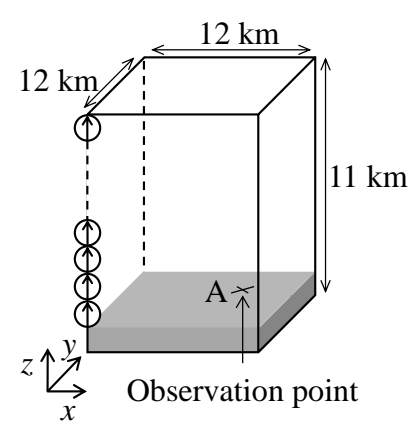

(a)

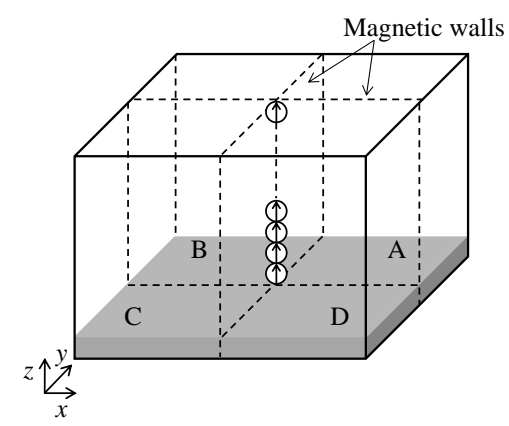

(b)

Figure 1. FDTD simulation model of a vertical lightning channel above flat, perfectly-conducting ground: (a) a single quadrant model with two magnetic walls; and (b) its equivalent four-quadrant model.

Figure 2 shows plan view of the configuration used for studying fields in the presence of a conducting (grounded) structure having a cross-section of $40 \mathrm{~m} \times 40 \mathrm{~m}$. The point at which the magnetic field is observed is located at $(x, y)=(7080 \mathrm{~m}, 7080 \mathrm{~m})$, which is $10,012 \mathrm{~m}(10.012 \mathrm{~km})$ away from the lightning channel and the azimuth angle is $45^{\circ}$ with respect to the $x$-axis (and $y$-axis) in the $x y$ plane. Also, computations were carried out for observation points at $(x, y)=(8960 \mathrm{~m}, 4480 \mathrm{~m})$ and $(10,000 \mathrm{~m}, 0 \mathrm{~m})$, which are $10,017 \mathrm{~m}$ and $10,000 \mathrm{~m}$, respectively, away from the lightning channel and the azimuth angles are $26.6^{\circ}\left(\tan ^{-1} \phi=0.5\right)$ and $0^{\circ}$ with respect to the $x$-axis and in the $x y$ plane. Note that the azimuth vector passes through the conducting structure diagonally for $\phi=45^{\circ}$, parallel to its walls for $\phi=0^{\circ}$ while it passes through the structure neither diagonally nor in parallel to its walls for $\phi=26.6^{\circ}$.

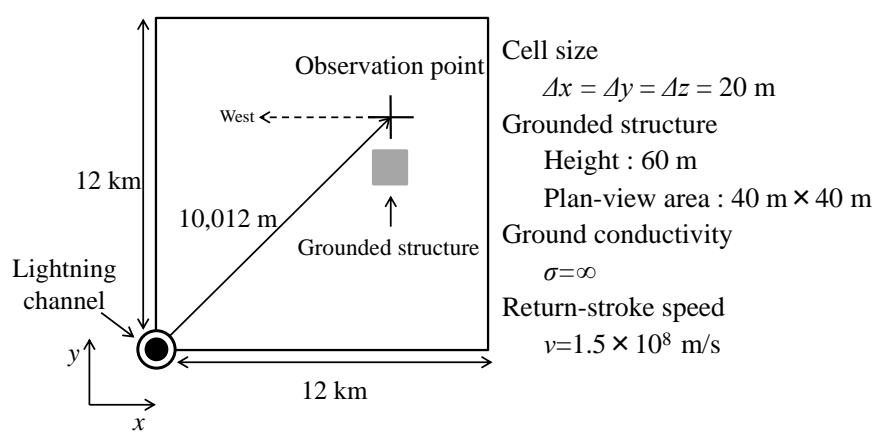

Figure 2. Plan view of the model for studying the influence of conducting structure (not to scale) on azimuth measured with respect to the west direction for the case of the true lightning azimuth $\phi=45^{\circ}$.

The conducting structure is represented by a perfectly-conducting rectangular parallelepiped of $40 \mathrm{~m} \times 40 \mathrm{~m} \times 60 \mathrm{~m}$, which is centered at different points near the magnetic-field observation point in quadrant A. Note that computations for structure conductivities of $0.1,1,10,100$ and $1000 \mathrm{mS} / \mathrm{m}$ were also performed. The total number of positions of the conducting structure was 24 , located within a square area of $200 \mathrm{~m} \times 200 \mathrm{~m}$, at the center of which $(7080 \mathrm{~m}, 7080 \mathrm{~m})$ the magnetic-field observation point was located for the case of azimuth angle equal to $45^{\circ}$ (this arrangement is clearly seen in the 
figures found in Section 3.2-Section 3.5). Identical conducting structures are present in quadrants B, C, and $D$, because of the symmetry due to the employed magnetic walls, as shown in Figure $1 b$. Since the structures in quadrants B, C, and D are far away from those in quadrant A and from the observation point, their influence on fields calculated in quadrant $\mathrm{A}$ is small.

The direction error $\Delta \phi$ in degrees for the true azimuth angle $\phi$ equal to $45^{\circ}$ is calculated from Equation (2) given below:

$$
\Delta \phi\left[^{\circ}\right]=\tan ^{-1}\left(\frac{H_{x}}{H_{y}}\right)-45^{\circ}
$$

where $H_{x}$ and $H_{y}$ are peak values of magnetic field in two orthogonal (east-west and south-north, respectively) directions at the observation point. The first term is the azimuth found from the ratio of $H_{x}$ to $H_{y}$, and the second term is the true azimuth for the configuration shown in Figure 2. Figure $3 a, b$ illustrate two cases with $\Delta \phi=0^{\circ}$ and $\Delta \phi=16^{\circ}$, respectively, for $\phi=45^{\circ}$. Note that magnetic fields in the west direction (negative $x$ direction) and in the north direction (positive $y$ direction) are each defined as positive.

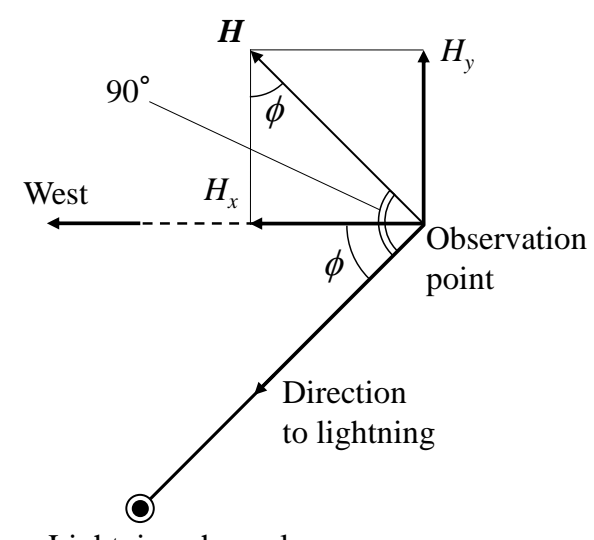

Lightning channel

$$
\phi=\tan ^{-1}\left(\frac{H_{x}}{H_{y}}\right)=45^{\circ}
$$$$
\Delta \phi=0
$$

(a)

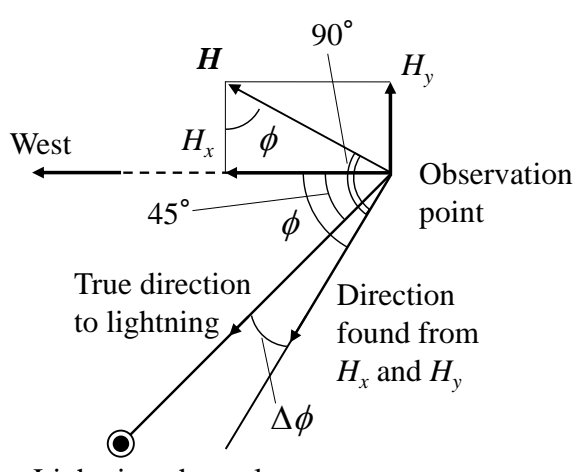

Lightning channel

$$
\begin{gathered}
\phi=\tan ^{-1}\left(\frac{H_{x}}{H_{y}}\right) \neq 45^{\circ} \\
\Delta \phi \neq 0
\end{gathered}
$$

(b)

Figure 3. Illustration of lightning direction finding from $H_{x}$ and $H_{y}$ for the case of the true lightning azimuth $\phi=45^{\circ}$ : (a) Ideal case of no azimuth error (direction found from $H_{x}$ and $H_{y}$ is the same as the true direction to lightning); (b) the case of the presence of $60-\mathrm{m}$ tall conducting structure at $(7080,7040)$ (see Table 1), which causes an azimuth error $\Delta \phi=16$.

\section{Analysis and Results}

\subsection{Testing the Model Validity}

In order to investigate the validity of our FDTD simulation, magnetic field due to a lightning strike to a flat ground without conducting structure is computed, and the FDTD-computed field is compared with the corresponding one predicted by the exact magnetic field equation [10].

Figure 4 shows FDTD-computed waveforms of $H_{x}$ and $H_{y}$ at an observation point $(7080 \mathrm{~m}, 7080 \mathrm{~m})$ for a lightning strike to a flat perfectly-conducting ground. The solid line shows $H_{x}$, and the broken line shows $H_{y}$. The waveform of $H_{x}$ is indistinguishable from the waveform of $H_{y}$, because the lightning azimuth angle (measured with respect to West) is equal to $45^{\circ}$.

Figure 5 shows the FDTD-computed waveform of total magnetic field $\left(H_{x}{ }^{2}+H_{y}{ }^{2}\right)^{1 / 2}$ at the observation point $(7080 \mathrm{~m}, 7080 \mathrm{~m})$, and the corresponding waveform computed using Uman et al.'s [10] exact equation, which is given below: 


$$
H_{\varphi}(D, t)=\frac{1}{2 \pi}\left[\int_{0}^{L^{\prime}(t)} \frac{\sin \theta}{R^{2}} I\left(z^{\prime}, t-R / c\right) d z^{\prime}+\int_{0}^{L^{\prime}(t)} \frac{\sin \theta}{c R} \frac{\partial I\left(z^{\prime}, t-R / c\right)}{\partial t} d z^{\prime}\right]
$$

where $D$ is the horizontal distance from the lightning channel to the observation point, $L^{\prime}(t)$ is the height of the return-stroke wavefront as seen by the observer at time $t, R$ is the inclined distance from the channel segment at height $z^{\prime}$ to the observation point equal to $\left(D^{2}+z^{\prime 2}\right)^{1 / 2}, \theta$ is the angle between the $z$-axis and inclined distance vector, $I\left(z^{\prime}, t\right)$ is the current along the channel at height $z^{\prime}$ and time $t$, and $c$ is the speed of light.

The solid line in Figure 5 represents the field computed using the FDTD method and the broken line the field computed using Equation (3). The FDTD-computed waveform agrees well with the corresponding one based on exact Equation (3), both for the case of TL model. It is clear from the above comparison that our FDTD simulation yields reasonably accurate results.

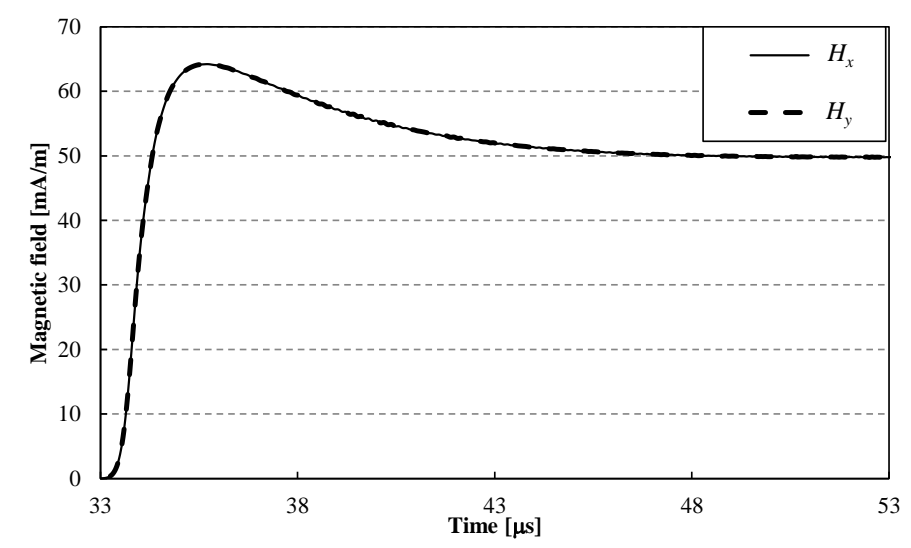

Figure 4. FDTD-computed waveforms of $H_{x}$ and $H_{y}$ at an observation point at $(7080 \mathrm{~m}, 7080 \mathrm{~m})$ for a lightning strike to a flat, perfectly-conducting ground (TL model, $v=150 \mathrm{~m} / \mu \mathrm{s}$ ).

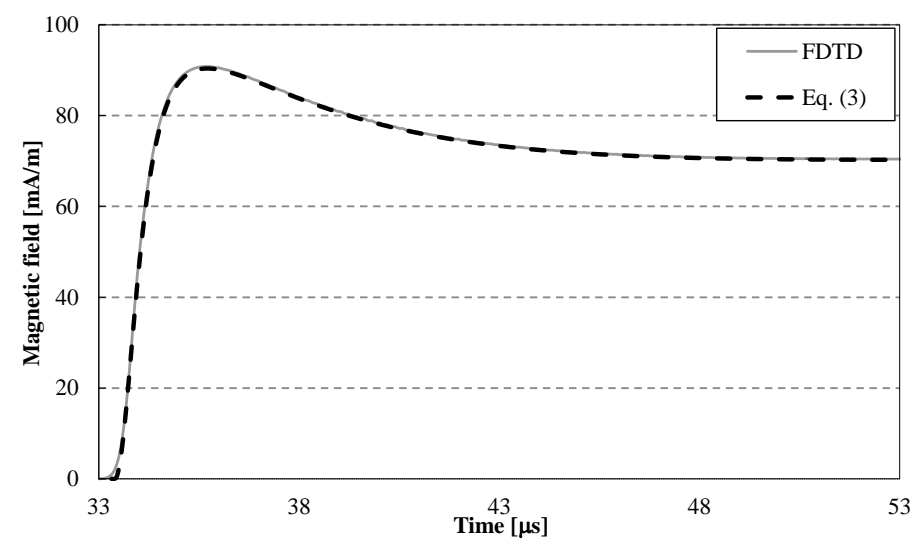

Figure 5. FDTD-computed waveform of total azimuthal magnetic field at an observation point located at $(7080 \mathrm{~m}, 7080 \mathrm{~m})$ and the corresponding waveform computed using Equation (3) (TL model, $v=150 \mathrm{~m} / \mu \mathrm{s})$.

\subsection{Influence of the Presence of Conducting Structure}

Table 1 shows FDTD-computed peak values of $H_{x}$ and $H_{y}$ and the azimuth errors in degrees due to the presence of a nearby conducting structure having a height of $60 \mathrm{~m}$ and a square cross-section of $40 \mathrm{~m} \times 40 \mathrm{~m}$ estimated from the ratio of $H_{x}$ to $H_{y}$ peaks. The results are computed for a current risetime equal to $1 \mu \mathrm{s}$. In this computation, the ground conductivity is set to $\infty$. Error in the counterclockwise 
direction relative to the correct azimuth of $\phi=45^{\circ}$ (see Figure 2) is defined as positive. Figure 6 shows the color-coded plot of azimuth error due to the presence of nearby conducting structure. In Figure 6, the darker the shading, the larger the error.

When the conducting structure is symmetrically located on the straight line that connects the lightning channel with the observation point (in other words, the azimuth vector passes through the conducting structure diagonally), its presence equally influences $H_{x}$ and $H_{y}$. Therefore, no azimuth error occurs. When the conducting structure is not located on that line, its influence is greater when it is located closer to the observation point. If the distance from the structure to the observation point is the same, the structure behind the observation point is more significant than that in front of it. Note that the azimuth error is zero when two identical structures are located symmetrically with respect to the direction to lightning, for example at $(7040,7080)$ and at $(7080,7040)$, since positive and negative errors compensate each other.
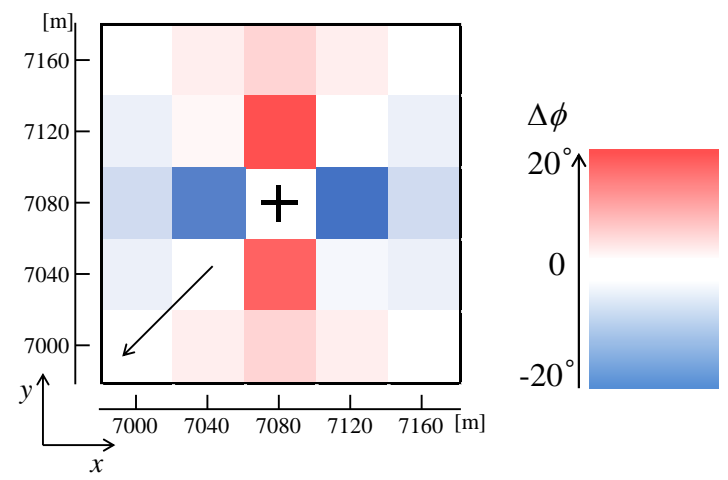

Figure 6. Color-coded azimuth error (in degrees) due to the presence of a nearby conducting structure having a height of $60 \mathrm{~m}$ and a square cross-section of $40 \mathrm{~m} \times 40 \mathrm{~m}$ for the case of true azimuth $\phi$ equal to $45^{\circ}$. Each small square, except for the one in the center, represents the position of conducting structure, with the total number of such positions being 24 . The large plus sign in the center ' + ' indicates the location of observation point, and the arrow indicates the true direction to lightning.

Table 1. FDTD-computed peak values of $H_{x}$ and $H_{y}$, and azimuth errors estimated from Equation (2) for the case of true azimuth $\phi$ equal to $45^{\circ}$ and current risetime equal to $1 \mu \mathrm{s}$.

\begin{tabular}{cccc}
\hline Position of Structure Center & $\boldsymbol{H}_{\boldsymbol{x}}(\mathbf{m A} / \mathbf{m})$ & $\boldsymbol{H}_{\boldsymbol{y}}(\mathbf{m A} / \mathbf{m})$ & $\boldsymbol{\Delta} \boldsymbol{\phi}\left(^{\circ}\right)$ \\
\hline$(7000,7000)$ & 66 & 66 & 0 \\
$(7000,7040)$ & 64 & 69 & 2 \\
$(7000,7080)$ & 58 & 68 & -5 \\
$(7000,7120)$ & 59 & 62 & -2 \\
$(7000,7160)$ & 62 & 62 & 0 \\
$(7040,7000)$ & 69 & 64 & 2 \\
$(7040,7040)$ & 74 & 74 & 0 \\
$(7040,7080)$ & 38 & 76 & -18 \\
$(7040,7120)$ & 54 & 53 & 1 \\
$(7040,7160)$ & 63 & 59 & 2 \\
$(7080,7000)$ & 68 & 58 & 5 \\
$(7080,7040)$ & 76 & 38 & 18 \\
$(7080,7120)$ & 80 & 38 & 20 \\
$(7080,7160)$ & 69 & 58 & 5 \\
$(7120,7000)$ & 62 & 59 & 2 \\
$(7120,7040)$ & 53 & 54 & -1 \\
$(7120,7080)$ & 38 & 80 & -20 \\
$(7120,7120)$ & 74 & 74 & 0 \\
$(7120,7160)$ & 70 & 64 & 2 \\
$(7160,7000)$ & 62 & 62 & 0 \\
$(7160,7040)$ & 59 & 63 & -2 \\
$(7160,7080)$ & 58 & 69 & -5 \\
$(7160,7120)$ & 64 & 70 & -2 \\
$(7160,7160)$ & 66 & 66 & 0 \\
\hline
\end{tabular}




\subsection{Influence of Lightning Current Risetime}

Tables 2 and 3 show FDTD-computed peak values of $H_{x}$ and $H_{y}$ peaks and the direction errors for true azimuth $\phi$ equal to $45^{\circ}$ and current risetimes of 0.5 and $3 \mu \mathrm{s}$, respectively. Figure $7 \mathrm{a}, \mathrm{b}$ show the corresponding plots of color-coded azimuth error. In these computations, the ground conductivity is set to $\infty$. It appears from comparison of Tables 1 and 3 or Figures 6 and $7 \mathrm{~b}$ that the change from 1 to $3 \mu \mathrm{s}$ in current risetime only slightly influences the azimuth error. When the current risetime is $0.5 \mu \mathrm{s}$, the azimuth error due to the presence of nearby conducting structure is larger.

Table 2. FDTD-computed peak values of $H_{x}$ and $H_{y}$, and azimuth errors estimated from Equation (2) for the case of true azimuth $\phi$ equal to $45^{\circ}$ and current risetime equal to $0.5 \mu \mathrm{s}$.

\begin{tabular}{|c|c|c|c|}
\hline Position of Structure Center & $H_{x}(\mathrm{~mA} / \mathrm{m})$ & $H_{y}(\mathrm{~mA} / \mathrm{m})$ & $\Delta \phi\left(^{\circ}\right)$ \\
\hline$(7000,7000)$ & 69 & 69 & 0 \\
\hline$(7000,7040)$ & 67 & 76 & -4 \\
\hline$(7000,7080)$ & 57 & 77 & -8 \\
\hline$(7000,7120)$ & 61 & 69 & -4 \\
\hline$(7000,7160)$ & 67 & 66 & 0 \\
\hline$(7040,7000)$ & 76 & 67 & 4 \\
\hline$(7040,7040)$ & 81 & 81 & 0 \\
\hline$(7040,7080)$ & 37 & 90 & -22 \\
\hline$(7040,7120)$ & 72 & 62 & 4 \\
\hline$(7040,7160)$ & 75 & 62 & 5 \\
\hline$(7080,7000)$ & 77 & 57 & 8 \\
\hline$(7080,7040)$ & 90 & 37 & 22 \\
\hline$(7080,7120)$ & 113 & 38 & 27 \\
\hline$(7080,7160)$ & 86 & 57 & 11 \\
\hline$(7120,7000)$ & 69 & 61 & 4 \\
\hline$(7120,7040)$ & 62 & 72 & -4 \\
\hline$(7120,7080)$ & 38 & 113 & -27 \\
\hline$(7120,7120)$ & 92 & 92 & 0 \\
\hline$(7120,7160)$ & 84 & 70 & 5 \\
\hline$(7160,7000)$ & 66 & 67 & 0 \\
\hline$(7160,7040)$ & 62 & 75 & -5 \\
\hline$(7160,7080)$ & 57 & 86 & -11 \\
\hline$(7160,7120)$ & 70 & 84 & -5 \\
\hline$(7160,7160)$ & 75 & 75 & 0 \\
\hline
\end{tabular}

Table 3. FDTD-computed peak values of $H_{x}$ and $H_{y}$, and azimuth errors estimated from Equation (2) for the case of true azimuth $\phi$ equal to $45^{\circ}$ and current risetime equal to $3 \mu \mathrm{s}$.

\begin{tabular}{cccc}
\hline Position of Structure Center & $\boldsymbol{H}_{\boldsymbol{x}}(\mathbf{m A} / \mathbf{m})$ & $\boldsymbol{H}_{\boldsymbol{y}}(\mathbf{m A} \mathbf{m})$ & $\left.\boldsymbol{\Delta} \boldsymbol{\phi} \mathbf{(}^{\circ}\right)$ \\
\hline$(7000,7000)$ & 68 & 68 & 0 \\
$(7000,7040)$ & 66 & 72 & -2 \\
$(7000,7080)$ & 60 & 70 & -5 \\
$(7000,7120)$ & 61 & 65 & -2 \\
$(7000,7160)$ & 64 & 64 & 0 \\
$(7040,7000)$ & 72 & 66 & 2 \\
$(7040,7040)$ & 76 & 76 & 0 \\
$(7040,7080)$ & 39 & 78 & -18 \\
$(7040,7120)$ & 55 & 55 & 0 \\
$(7040,7160)$ & 65 & 61 & 2 \\
$(7080,7000)$ & 70 & 60 & 5 \\
$(7080,7040)$ & 78 & 39 & 18 \\
$(7080,7120)$ & 78 & 40 & 18 \\
$(7080,7160)$ & 70 & 60 & 4 \\
$(7120,7000)$ & 65 & 61 & 2 \\
$(7120,7040)$ & 55 & 55 & 0 \\
$(7120,7080)$ & 40 & 78 & -18 \\
$(7120,7120)$ & 76 & 76 & 0 \\
$(7120,7160)$ & 72 & 67 & 2 \\
$(7160,7000)$ & 64 & 64 & 0 \\
$(7160,7040)$ & 61 & 65 & -2 \\
$(7160,7080)$ & 60 & 70 & -4 \\
$(7160,7120)$ & 67 & 72 & -2 \\
$(7160,7160)$ & 68 & 68 & 0 \\
\hline
\end{tabular}




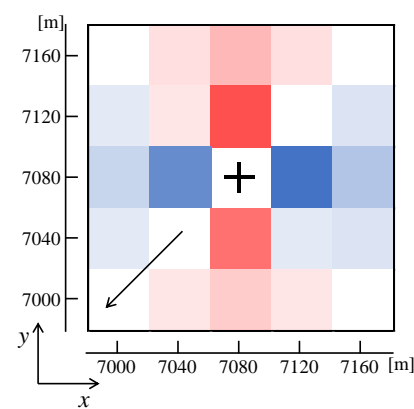

(a)
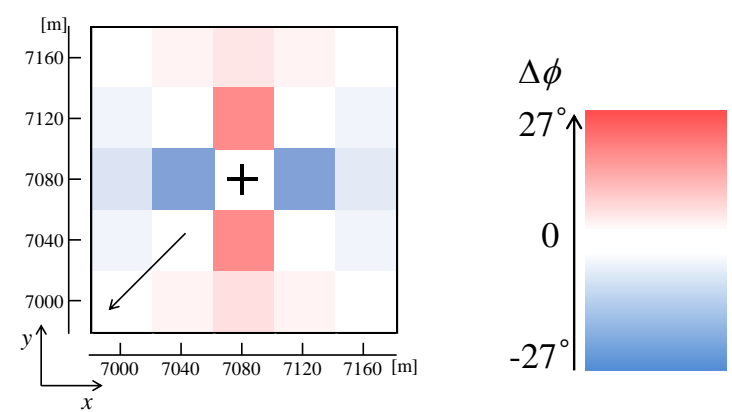

Figure 7. Color-coded azimuth error (in degrees) due to the presence of a nearby conducting structure having a height of $60 \mathrm{~m}$ and a square cross-section of $40 \mathrm{~m} \times 40 \mathrm{~m}$ for the case of true azimuth $\phi$ equal to $45^{\circ}$ and current risetimes equal to (a) $0.5 \mu$ s and (b) $3 \mu \mathrm{s}$.

\subsection{Influence of Ground Conductivity}

Table 4 shows FDTD-computed peak values of $H_{x}$ and $H_{y}$ and errors in azimuth estimated from Equation (1) for ground conductivity values equal to $0.1,1,5,10$ and $1000 \mathrm{mS} / \mathrm{m}$ and relative permittivity equal to 10 . The structure conductivity is set to $\infty$. The current risetime is set to $1 \mu \mathrm{s}$. Figure 8 shows plots of color-coded azimuth error due to the presence of nearby conducting structure for true azimuth $\phi$ equal to $45^{\circ}$ and ground conductivity values equal to $0.1,5$ and $1000 \mathrm{mS} / \mathrm{m}$. Figure 9 shows azimuth errors for the true azimuth $\phi$ equal to $45^{\circ}$ as a function of ground conductivity when the conducting structure is located at $(7000 \mathrm{~m}, 7000 \mathrm{~m}),(7000 \mathrm{~m}, 7080 \mathrm{~m}),(7040 \mathrm{~m}, 7080 \mathrm{~m}),(7120 \mathrm{~m}$, $7080 \mathrm{~m})$, and $(7160 \mathrm{~m}, 7080 \mathrm{~m})$.

It appears from these results that the azimuth error due to the presence of nearby conducting structure decreases with decreasing ground conductivity.

Table 4. FDTD-computed azimuth errors (in degrees) estimated from Equation (2) for true azimuth $\phi$ equal to $45^{\circ}$ and ground conductivity values equal to $0.1,1,5,10$ and $1000 \mathrm{mS} / \mathrm{m}$.

\begin{tabular}{|c|c|c|c|c|c|}
\hline \multirow{2}{*}{ Position of Structure Center } & \multicolumn{5}{|c|}{$\Delta \phi\left(^{\circ}\right)$} \\
\hline & $0.1 \mathrm{mS} / \mathrm{m}$ & $1 \mathrm{mS} / \mathrm{m}$ & $5 \mathrm{mS} / \mathrm{m}$ & $10 \mathrm{mS} / \mathrm{m}$ & $1000 \mathrm{mS} / \mathrm{m}$ \\
\hline$(7000,7000)$ & 0 & 0 & 0 & 0 & 0 \\
\hline$(7000,7040)$ & -1 & -2 & -2 & -2 & -2 \\
\hline$(7000,7080)$ & -3 & -4 & -5 & -5 & -5 \\
\hline$(7000,7120)$ & -1 & -1 & -2 & -2 & -2 \\
\hline$(7000,7160)$ & 0 & 0 & 0 & 0 & 0 \\
\hline$(7040,7000)$ & 1 & 2 & 2 & 2 & 2 \\
\hline$(7040,7040)$ & 0 & 0 & 0 & 0 & 0 \\
\hline$(7040,7080)$ & -13 & -16 & -18 & -18 & -18 \\
\hline$(7040,7120)$ & 0 & 0 & 0 & 0 & 1 \\
\hline$(7040,7160)$ & 1 & 1 & 2 & 2 & 2 \\
\hline$(7080,7000)$ & 3 & 4 & 5 & 5 & 5 \\
\hline$(7080,7040)$ & 13 & 16 & 18 & 18 & 18 \\
\hline$(7080,7120)$ & 14 & 16 & 18 & 19 & 20 \\
\hline$(7080,7160)$ & 3 & 4 & 5 & 5 & 5 \\
\hline$(7120,7000)$ & 1 & 1 & 2 & 2 & 2 \\
\hline$(7120,7040)$ & 0 & 0 & 0 & 0 & -1 \\
\hline$(7120,7080)$ & -14 & -16 & -18 & -19 & -20 \\
\hline$(7120,7120)$ & 0 & 0 & 0 & 0 & 0 \\
\hline$(7120,7160)$ & 1 & 2 & 2 & 2 & 2 \\
\hline$(7160,7000)$ & 0 & 0 & 0 & 0 & 0 \\
\hline$(7160,7040)$ & -1 & -1 & -2 & -2 & -2 \\
\hline$(7160,7080)$ & -3 & -4 & -5 & -5 & -5 \\
\hline$(7160,7120)$ & -1 & -2 & -2 & -2 & -2 \\
\hline$(7160,7160)$ & 0 & 0 & 0 & 0 & 0 \\
\hline
\end{tabular}




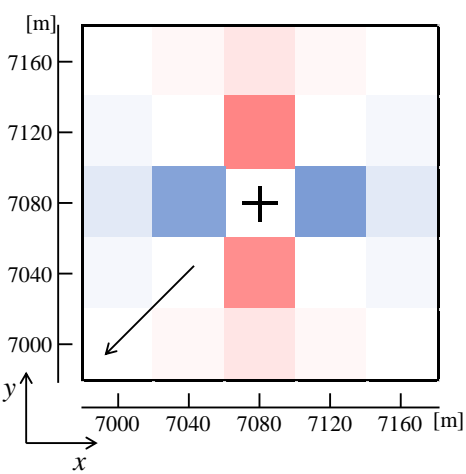

(a)

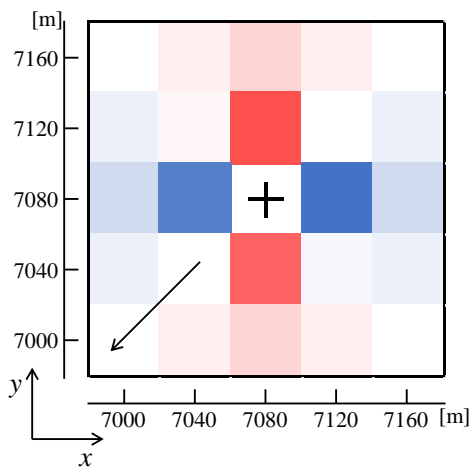

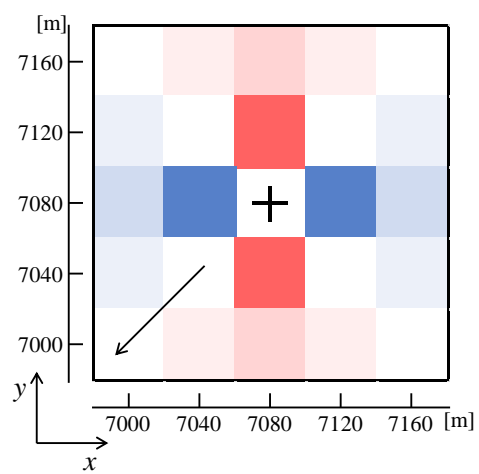

(b)

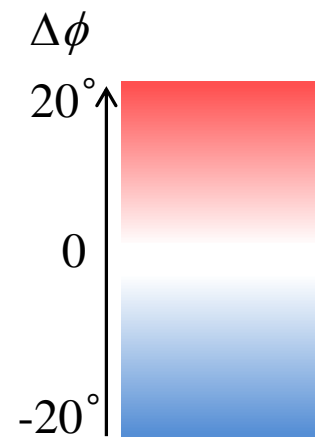

(c)

Figure 8. Color-coded azimuth error due to the presence of a nearby conducting structure having a height of $60 \mathrm{~m}$ and a square cross-section of $40 \mathrm{~m} \times 40 \mathrm{~m}$ for the case of true azimuth $\phi$ equal to $45^{\circ}$ and ground conductivity values equal to (a) $0.1 \mathrm{mS} / \mathrm{m}$; (b) $5 \mathrm{mS} / \mathrm{m}$; and (c) $1000 \mathrm{mS} / \mathrm{m}$.

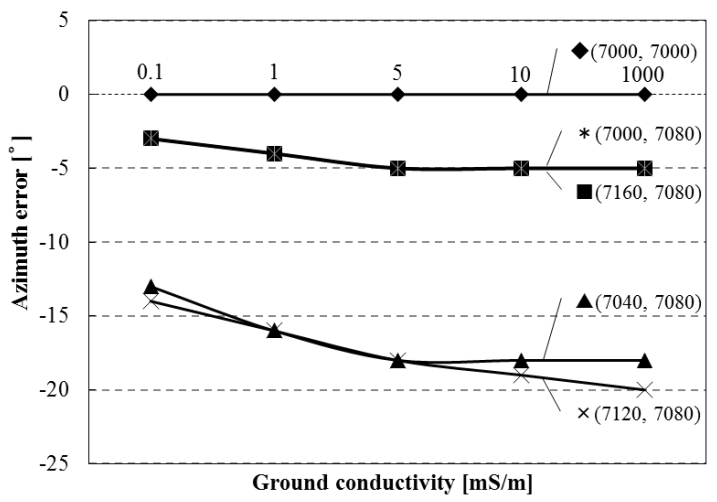

Figure 9. Azimuth errors as a function of ground conductivity when the conducting structure is located at $(7000 \mathrm{~m}, 7000 \mathrm{~m}),(7000 \mathrm{~m}, 7080 \mathrm{~m}),(7040 \mathrm{~m}, 7080 \mathrm{~m}),(7120 \mathrm{~m}, 7080 \mathrm{~m})$, and $(7160 \mathrm{~m}, 7080 \mathrm{~m})$.

\subsection{Influence of Grounded Structure Conductivity}

Table 5 shows azimuth errors estimated from Equation (2) for different values of structure conductivity equal to $0.1,1,10,100,1000 \mathrm{mS} / \mathrm{m}$ and relative permittivity equal to 10 . The ground conductivity is set to $\infty$. The current risetime is set to $1 \mu \mathrm{s}$. Figure 10 shows color-coded azimuth error due to the presence of nearby conducting structure for true azimuth $\phi$ equal to $45^{\circ}$ and different values of structure conductivity equal to $0.1,1,10,1000 \mathrm{mS} / \mathrm{m}$.

It appears from these results that as the structure conductivity decreases, the azimuth error becomes smaller, except for the cases that the structure is located at $(7040 \mathrm{~m}, 7080 \mathrm{~m})$ and $(7080 \mathrm{~m}$, 
$7040 \mathrm{~m}$ ). This is because the induced current in the conducting structure and the resultant scattered field decrease with decreasing the structure conductivity.

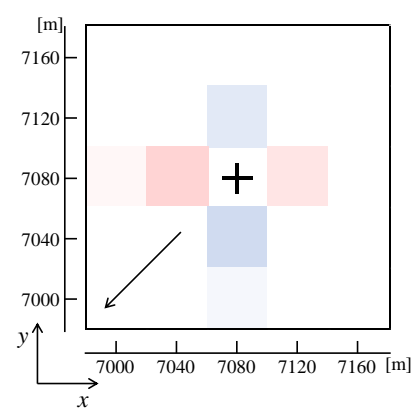

(a)

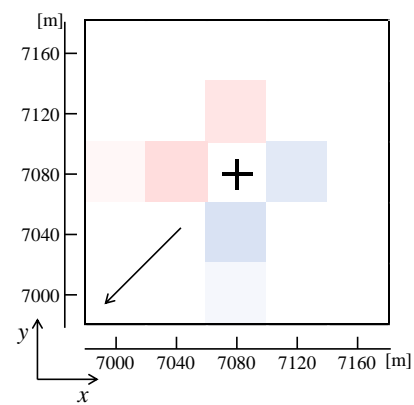

(b)

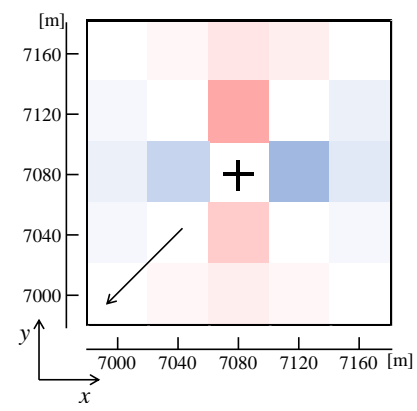

(c)

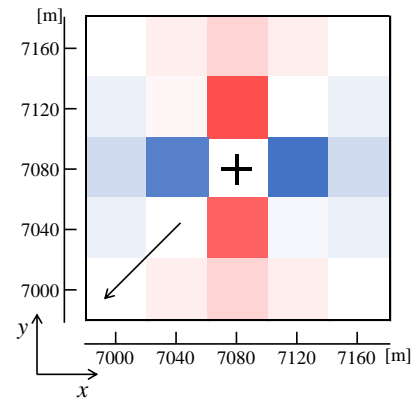

(d)

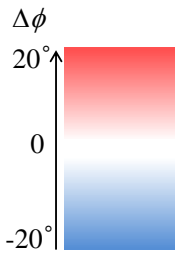

Figure 10. Color-coded azimuth error due to the presence of a nearby conducting structure having a height of $60 \mathrm{~m}$ and a square cross-section of $40 \mathrm{~m} \times 40 \mathrm{~m}$ for true azimuth $\phi$ equal to $45^{\circ}$ and different values of structure conductivity equall to (a) $0.1 \mathrm{mS} / \mathrm{m}$; (b) $1 \mathrm{mS} / \mathrm{m} ;$ (c) $10 \mathrm{mS} / \mathrm{m}$; and (d) $1000 \mathrm{mS} / \mathrm{m}$.

Table 5. Azimuth errors estimated from Equation (2) for true azimuth $\phi$ equal to $45^{\circ}$ and different values of structure conductivity equal to $0.1,1,10,100,1000 \mathrm{mS} / \mathrm{m}$.

\begin{tabular}{cccccc}
\hline \multirow{2}{*}{ Position of Structure Center } & \multicolumn{5}{c}{$\left.\boldsymbol{\Delta} \boldsymbol{\phi} \mathbf{(}^{\circ}\right)$} \\
\cline { 2 - 6 } & $\mathbf{0 . 1} \mathbf{~} \mathbf{S} / \mathbf{m}$ & $\mathbf{1} \mathbf{~} \mathbf{S} / \mathbf{m}$ & $\mathbf{5} \mathbf{~} \mathbf{S} / \mathbf{m}$ & $\mathbf{1 0} \mathbf{~} \mathbf{S} / \mathbf{m}$ & $\mathbf{1 0 0 0} \mathbf{~} \mathbf{S} / \mathbf{m}$ \\
\hline (7000, 7000) & 0 & 0 & 0 & 0 & 0 \\
$(7000,7040)$ & 0 & 0 & -1 & -1 & -2 \\
$(7000,7080)$ & 1 & 1 & -1 & -2 & -5 \\
$(7000,7120)$ & 0 & 0 & 0 & -1 & -2 \\
$(7000,7160)$ & 0 & 0 & 0 & 0 & 0 \\
$(7040,7000)$ & 0 & 0 & 1 & 1 & 2 \\
$(7040,7040)$ & 0 & 0 & 0 & 0 & 0 \\
$(7040,7080)$ & 5 & 4 & -3 & -6 & -18 \\
$(7040,7120)$ & 0 & 0 & 0 & 0 & 1 \\
$(7040,7160)$ & 0 & 0 & 1 & 1 & 2 \\
$(7080,7000)$ & -1 & -1 & 1 & 2 & 5 \\
$(7080,7040)$ & -5 & -4 & 3 & 6 & 18 \\
$(7080,7120)$ & -3 & 3 & 7 & 10 & 20 \\
$(7080,7160)$ & 0 & 0 & 2 & 3 & 5 \\
$(7120,7000)$ & 0 & 0 & 0 & 1 & 2 \\
$(7120,7040)$ & 0 & 0 & 0 & 0 & -1 \\
$(7120,7080)$ & 3 & -3 & -7 & -10 & -20 \\
$(7120,7120)$ & 0 & 0 & 0 & 0 & 0 \\
$(7120,7160)$ & 0 & 0 & 1 & 2 & 2 \\
$(7160,7000)$ & 0 & 0 & 0 & 0 & 0 \\
$(7160,7040)$ & 0 & 0 & -1 & -1 & -2 \\
$(7160,7080)$ & 0 & 0 & -2 & -3 & -5 \\
$(7160,7120)$ & 0 & 0 & -1 & -2 & -2 \\
$(7160,7160)$ & 0 & 0 & 0 & 0 & 0 \\
\hline
\end{tabular}




\subsection{Influence of Structure Location Relative to the Azimuth Vector}

Table 6 shows azimuth errors estimated for the observation point at $(x, y)=(8960 \mathrm{~m}, 4480 \mathrm{~m})$, which is $10,017 \mathrm{~m}$ away from the lightning channel and the azimuth angle is $26.6^{\circ}\left(\tan ^{-1} \phi=0.5\right)$ with respect to the $x$-axis (- $x$ direction) in the $x y$ plane. The conductivity of both the structure and ground is set to $\infty$. The current risetime is set to $1 \mu \mathrm{s}$. Figure 11 shows color-coded azimuth error due to the presence of nearby conducting structure for true azimuth $\phi$ equal to $26.6^{\circ}$.

It appears from these results that an azimuth error occurs even when the center of the conducting structure is located on the straight line that connects the lightning channel and the observation point. This is because the conducting structure having a square cross-section is not symmetrically located on that line (in other words, the azimuth vector does not pass through the conducting structure diagonally) as shown in Figure 12a. Note that for $\phi=45^{\circ}$ no azimuth error occurs since the azimuth vector passes through the conducting structure diagonally, as shown in Figure 12b, so that the structure equally influences $H_{x}$ and $H_{y}$ and $H_{x} / H_{y}$ is the same as in the absence of structure. Also note that for $\phi=0^{\circ}$ no azimuth error occurs since the azimuth vector is parallel to the walls of the structure as shown in Figure 12c. This is confirmed by the 3D FDTD simulation, although the results are not shown here.

Table 6. Azimuth errors estimated for true azimuth $\phi$ equal to $26.6^{\circ}\left(\tan ^{-1} \phi=0.5\right)$.

\begin{tabular}{cccc}
\hline Position of Structure Center & $\boldsymbol{H}_{\boldsymbol{x}} \mathbf{( \mathbf { m A } / \mathbf { m } )}$ & $\boldsymbol{H}_{\boldsymbol{y}} \mathbf{( \mathbf { m A } / \mathbf { m } )}$ & $\left.\boldsymbol{\Delta} \boldsymbol{\phi} \mathbf{(}^{\circ}\right)$ \\
\hline$(8880,4400)$ & 43 & 82 & 1 \\
$(8880,4440)$ & 42 & 86 & 0 \\
$(8880,4480)$ & 36 & 86 & -4 \\
$(8880,4520)$ & 36 & 81 & -3 \\
$(8920,4420)$ & 50 & 81 & 5 \\
$(8920,4460)$ & 41 & 112 & -6 \\
$(8920,4500)$ & 22 & 83 & -12 \\
$(8920,4540)$ & 36 & 74 & -1 \\
$(8960,4400)$ & 43 & 73 & 4 \\
$(8960,4440)$ & 49 & 48 & 19 \\
$(8960,4520)$ & 60 & 48 & 25 \\
$(8960,4560)$ & 47 & 73 & 6 \\
$(9000,4420)$ & 35 & 75 & -2 \\
$(9000,4460)$ & 23 & 89 & -12 \\
$(9000,4500)$ & 42 & 113 & -6 \\
$(9000,4540)$ & 52 & 81 & 6 \\
$(9040,4440)$ & 36 & 82 & -3 \\
$(9040,4480)$ & 36 & 87 & -4 \\
$(9040,4520)$ & 42 & 86 & 0 \\
$(9040,4560)$ & 44 & 83 & 2 \\
\hline
\end{tabular}
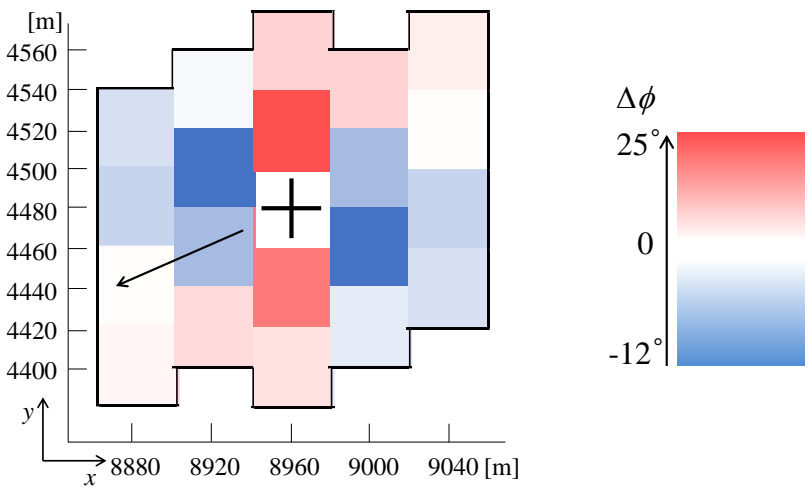

Figure 11. Color-coded azimuth error due to the presence of a nearby conducting structure having a height of $60 \mathrm{~m}$ and a square cross-section of $40 \mathrm{~m} \times 40 \mathrm{~m}$ for the case of true azimuth $\phi$ equal to $26.6^{\circ}$ $\left(\tan ^{-1} \phi=0.5\right)$. 


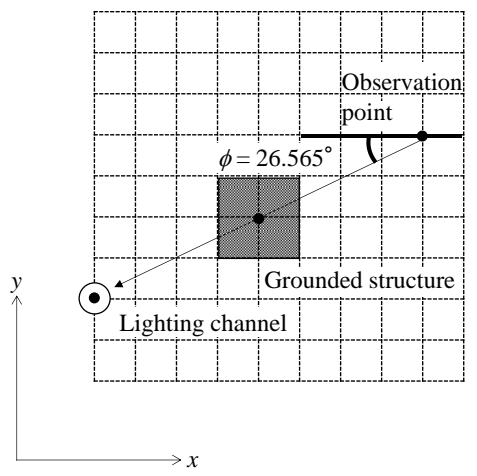

(a)

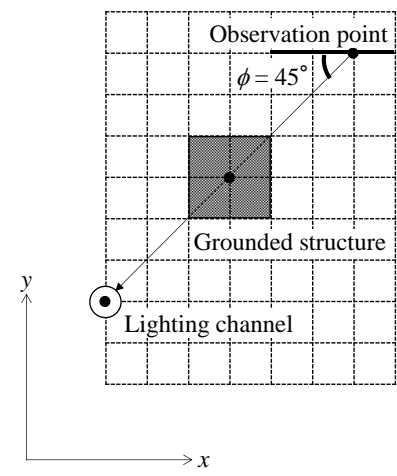

(b)

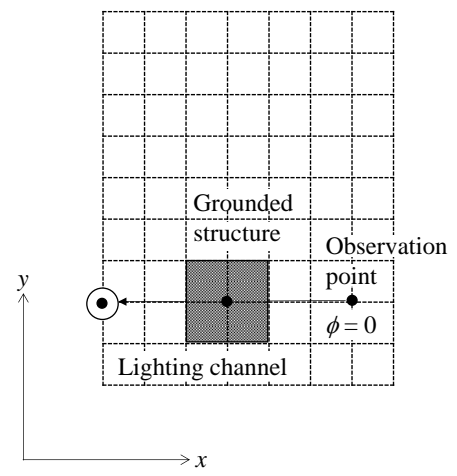

(c)

Figure 12. Illustration of locations of lightning channel, conducting structure, and observation point when the center of the structure is located on the straight line that connects the lightning channel with the observation point: (a) true azimuth $\phi$ is $26.6^{\circ}$; (b) true azimuth $\phi$ is $45^{\circ}$; (c) true azimuth $\phi$ is $0^{\circ}$.

\section{Conclusions}

In this paper, lightning electromagnetic fields in the presence of conducting structure having a height of $60 \mathrm{~m}$ and a square cross-section of $40 \mathrm{~m} \times 40 \mathrm{~m}$ within about $100 \mathrm{~m}$ of the field point have been analyzed using the 3D FDTD method. Influence of the structure on the two orthogonal components of magnetic field, $H_{x}$ and $H_{y}$, has been analyzed, and the resultant error in the estimated lightning azimuth has been studied. When the azimuth vector passes through the center of conducting structure diagonally (e.g., azimuth angle is $45^{\circ}$ ) or parallel to its walls (e.g., azimuth angle is $0^{\circ}$ ), the presence of structure equally influences $H_{x}$ and $H_{y}$, so that $H_{x} / H_{y}$ is the same as in the absence of structure. As a result, no azimuth error occurs in those configurations. When the structure is not located on that line, its influence is greater when it is located closer to the observation point. If the distance from the structure to the observation point is the same, the influence of structure behind the observation point is more significant than that in front of it. The azimuth error due to the presence of structure depends on the risetime of lightning current. When the current risetime is $0.5 \mu \mathrm{s}$, the azimuth error is generally larger than those for risetimes equal to 1 or $3 \mu \mathrm{s}$, except for some specific locations. The azimuth error due to the presence of structure decreases with decreasing ground conductivity. As the structure conductivity decreases, the azimuth error becomes smaller.

Acknowledgments: This work is supported in part by the Japan Society for the Promotion of Science (Grant number 15K05961). Vladimir A. Rakov was supported in part by the U.S. National Science Foundation and GRF grant 14.B25.31.0023. The data for this paper are available on request from the corresponding author.

Author Contributions: Yosuke Suzuki, Shohei Araki, Yoshihiro Baba, Toshihiro Tsuboi, Shigemitsu Okabe, and Vladimir A. Rakov performed the modeling and analysis. Yosuke Suzuki, Shohei Araki, Yoshihiro Baba, and Vladimir A. Rakov wrote the paper.

Conflicts of Interest: The authors declare no conflict of interest.

\section{Abbreviations}

FDTD finite-difference time-domain

LLS lightning locating system

NLDN National Lightning Detection Network

TL transmission line

\section{References}

1. Rakov, V.A.; Uman, M.A. Lightning: Physics and Effects; Cambridge University Press: Cambridge, UK, 2003.

2. Horner, F. The accuracy of the location sources of atmospherics by radio direction finding. Proc. IEE-III Radio Commun. Eng. 1954, 101, 383-390. [CrossRef] 
3. Horner, F. Very-low-frequency propagation and direction finding. Proc. IEE-B Radio Electron. Eng. 1957, 101, 73-80. [CrossRef]

4. Nishino, M.; Iwai, A.; Kashiwagi, M. Location of the sources of atmospherics in and around Japan. In Proceedings of the Research Institute Atmospherics, Nagoya University, Aichi, Japan, 30 March 1973; Volume 20, pp. 9-21.

5. Kidder, R.E. The location of lightning flashes at ranges less than $100 \mathrm{~km}$. J. Atmos. Terr. Phys. 1973, 35, 283-290. [CrossRef]

6. Krider, E.P.; Noggle, R.C.; Uman, M.A. A gated wideband magnetic direction finder for lightning return strokes. J. Appl. Meteorol. 1976, 15, 301-306. [CrossRef]

7. Yee, K.S. Numerical solution of initial boundary value problems involving Maxwell's equations in isotropic media. IEEE Trans. Antennas Propag. 1966, 14, 302-307.

8. Liao, Z.P.; Wong, H.L.; Yung, B.P.; Yuan, Y.F. A transmitting boundary for transient wave analysis. Sci. Sin. Ser. A 1984, 27, 1063-1076.

9. Baba, Y.; Rakov, V.A. On the transmission line model for lightning return stroke representation. Geophys. Res. Lett. 2003, 30. [CrossRef]

10. Uman, M.A.; McLain, D.K.; Krider, E.P. The electromagnetic radiation from a finite antenna. Am. J. Phys. 1975, 43, 33-38. [CrossRef]

11. Heidler, F. Traveling current source model for LEMP calculation. In Proceedings of the 6th International Zurich Symposium on Electromagnetic Compatibility, Zurich, Switzerland, 5 March 1985; pp. 157-162.

(C) 2016 by the authors; licensee MDPI, Basel, Switzerland. This article is an open access article distributed under the terms and conditions of the Creative Commons Attribution (CC-BY) license (http:/ / creativecommons.org/licenses/by/4.0/). 